\title{
ATLAS LAr Calorimeter Performance in LHC Run 2
}

\author{
M. Spalla ${ }^{a}$ on behalf of ATLAS Liquid Argon Group \\ ${ }^{a}$ Max-Planck-Institut für Physik \\ (Werner-Heisenberg-Institut), \\ München, Germany \\ E-mail: margherita.spalla@cern.ch
}

AвSTRACT: Liquid argon (LAr) sampling calorimeters are employed by ATLAS for all electromagnetic calorimetry and for hadronic and forward calorimetry in the region $1.5<|\eta|<4.9$. In the first LHC run a total luminosity of $27 \mathrm{fb}^{-1}$ has been collected at center-of-mass energies of 7-8 $\mathrm{TeV}$. After detector consolidation during a long shutdown, Run-2 started in 2015 and $139 \mathrm{fb}^{-1}$ of data at a center-of-mass energy of $13 \mathrm{TeV}$ have been recorded. In order to accomplish the Level-1 acceptance rate of $100 \mathrm{kHz}$ in Run-2 data taking, the number of read-out samples recorded and used for the energy and the time measurement has been modified from five to four while keeping the expected performance.

The well calibrated and highly granular LAr Calorimeter reached its design goals, both in terms of energy and direction measurement.

This contribution gives an overview of the detector operation, hardware improvements, monitoring and data quality procedures, as well as the achieved performance, including the calibration and stability of the electromagnetic scale, noise level, response uniformity and time resolution.

Keywords: Performance of High Energy Physics Detectors, Noble liquid detectors (scintillation, ionization, double-phase) 


\section{Contents}

1 Introduction 1

2 The ATLAS Liquid Argon Calorimeter 1

3 Performance 2

3.1 Time resolution 2

3.2 Energy resolution 2

4 Pulse shape measurement 3

5 LAr operation and data quality 4

5.1 Noise bursts 5

6 Conclusion 6

\section{Introduction}

The Large Hadron Collider (LHC) Run 2 has taken place between 2015 and 2018. The ATLAS detector has recorded $139 \mathrm{fb}^{-1}$ of proton-proton collision data, at $\sqrt{s}=13 \mathrm{TeV}$ and $25 \mathrm{~ns}$ bunch spacing, with about $89 \%$ data taking efficiency [1]. The number of interactions per bunch crossing has been 33.7 on average, reaching a maximum of 50-60 [1]. A highly performant and reliable calorimeter system plays an essential role in supporting the vast ATLAS physics program, as most analysis rely on precise energy measurement of physical objects such as electrons, photons, jets, missing transverse momentum, and others.

\section{The ATLAS Liquid Argon Calorimeter}

The Liquid Argon (LAr) calorimeter system is composed of multiple sampling calorimeters, all using liquid argon as active material. They are the Electromagnetic calorimeter, the Hadronic End-Cap and the Forward calorimeter. A complete description can be found in [2].

The Elecromagnetic calorimeter covers the barrel region $(|\eta|<1.475)$ as well as the two endcaps $(1.375<|\eta|<3.2)$. Lead is used as passive material and electrodes are built with an accordion shape for best azimuthal angle $(\phi)$ uniformity. The Hadronic End-Cap covers the area $1.5<|\eta|<3.2$ and the passive material consists of parallel copper plates. The Forward calorimeter is located at $3.1<|\eta|<4.9$ : it is made up of a copper or tungsten ${ }^{1}$ matrix: cylindrical holes are arranged into a honeycomb pattern, with the liquid argon filling the gaps between the rods and the holes' walls.

\footnotetext{
${ }^{1}$ The material depends on the layer.
} 
All calorimeters are segmented into multiple layers in the longitudinal shower direction.

The readout of the LAr Calorimeter is built of a Front-End and a Back-End part. The analog signal is amplified into 3 gains, shaped, sampled at $40 \mathrm{MHz}$ and stored in an analog buffer waiting for the Level-1 Trigger decision.

The calorimeter signals are also used at Trigger level. The analog sum of all layers contributing to a given $\eta \times \phi$ bin forms a trigger tower, towers are then sent to the Level-1 trigger central processor. If a Level-1 accept is received, the proper gain is selected and 4 samples are digitised and sent to the Back-End via optical links. The number of digitised samples has been changed from 5 to 4 after Run 1, in order to mantain the Level-1 acceptance rate of $100 \mathrm{kHz}$ despite the increased occupancy. At the Back-End, the digitised samples are combined into an energy measurement, using a set of optimal filtering coefficients. The energy is then converted from ADC counts to $\mathrm{MeV}$ through multiple calibration constants. Calibration runs have been recorded on a regular basis during the data taking period, in order to provide a stable energy response. The signal timing and a quality factor are also computed at this stage.

\section{Performance}

\subsection{Time resolution}

LAr Calorimeter timing has multiple applications, including rejection of Out Of Time PileUp ${ }^{2}$ and searches for long lived neutral particles ${ }^{3}$.

To help maintaining a good time resolution, the timing of Front-End Boards (FEBs) is synchronised using data from both, beam splashes and early collision runs. An example of the average time offset per FEB in the LAr Barrel region is shown in Figure 1a.

The LAr Calorimeter time resolution has been measured using $3.3 \mathrm{fb}^{-1}$ of collision data taken in 2015 at $13 \mathrm{TeV}$.

The data have been calibrated with a 7 step procedure as explained in [3]. Corrections have been derived with a $W \rightarrow e v$ data set and applied to an independent $Z \rightarrow e e$ data set to determine the resolution.

The resulting time resolution, expressed as a function of energy as $\sigma_{t}=\frac{p_{0}}{E} \oplus p_{1}$, is shown in Figure $1 \mathrm{~b}$ for the Electromagnetic Barrel calorimeter. The constant term $\left(p_{1}\right)$ amounts to 230-260 ps depending on the gain and includes a contribution of roughly 200 ps due to the beam spread.

\subsection{Energy resolution}

The performance of the LAr Calorimeter energy response can be inferred from measurements of the energy scale and resolution of electrons and photons in ATLAS. Recent Run 2 results can be found in [4].

The resolution is evaluated as a function of energy $\left(\frac{\sigma_{E}}{E}=\frac{a}{\sqrt{E}} \oplus \frac{b}{E} \oplus c\right)$, using MonteCarlo simulation. An additional contribution to the constant term is then derived by comparing with $Z \rightarrow e e$ data. This term (labelled $c_{i}$ ) is shown in Figure 2a, it amounts to less than $1 \%$ in the Barrel. In the same

\footnotetext{
${ }^{2}$ Energy deposits from collisions in the same bunch crossing as the one of interest are referred to as In Time PileUp. Those from different bunch crossings are called Out Of Time PileUp.

${ }^{3}$ Whose detection involves delayed signals in the calorimeters.
} 


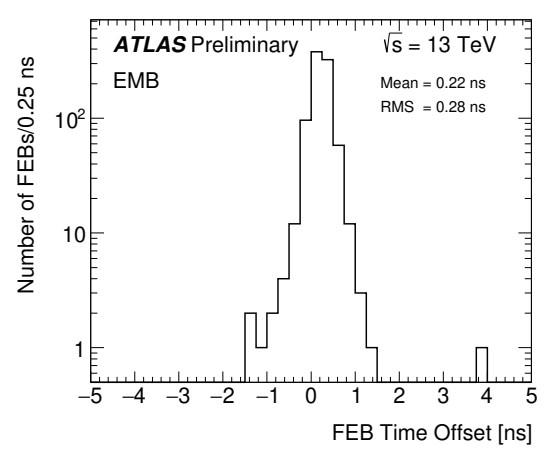

(a)

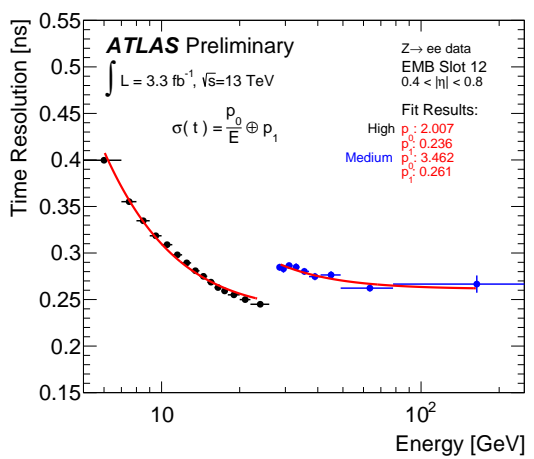

(b)

Figure 1: a) Average time per Front End Board (FEB) in the LAr Electromagnetic Barrel (EMB) with $1.6 \mathrm{fb}^{-1}$ of 2015 collision data [3]. b) Time resolution as a function of energy for High and Medium gain in Electromagnetic Barrel, Slot $12(0.4<|\eta|<0.8)$ [3].

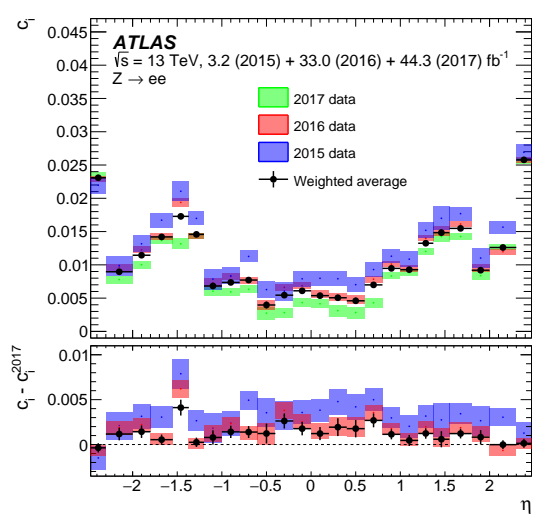

(a)

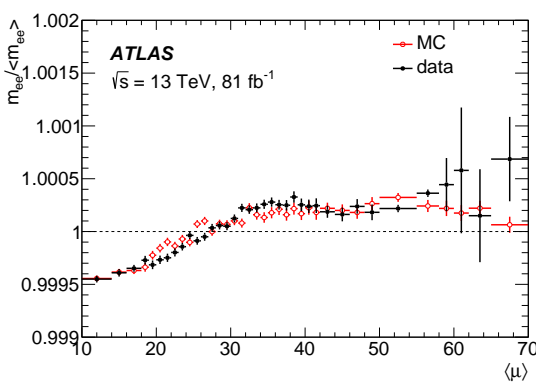

(b)

Figure 2: a) Data-driven contribution to the constant term in energy resolution ( $c_{i}$, where $i$ refers to the $\eta$ region the term applies to), as a function of $\eta$ [4]. The shaded areas represent the statistical uncertainties. b) Relative variation of the reconstructed dielectron mass peak in $Z \rightarrow e e$ events as a function of the average number of interactions per bunch crossing [4]. The error bars represent the statistical uncertainties.

region the contribution to the constant term evaluated from MonteCarlo is roughly half a percent. The stability of the electron energy scale with PileUp has also been evaluated. It is shown in Figure $2 b$, as a function of the average number of interactions per bunch crossing. The overall variation remains within half a permille, the visible dependence has been attributed to the electron reconstruction algorithm rather than the calorimeter.

\section{Pulse shape measurement}

Data recorded in runs with a few colliding bunches have been used to precisely measure the LAr analog pulse shape, such runs have been repeated in 2016, 2017 and 2018. 


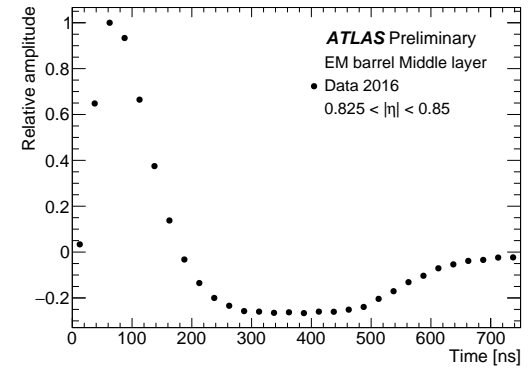

(a)

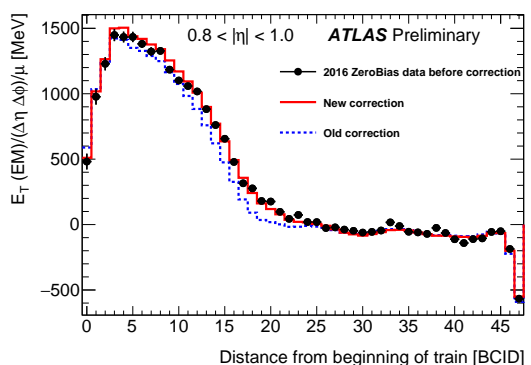

(b)

Figure 3: a) Typical ionization pulse shapes in the EM barrel middle layer [3]. b) Average transverse energy per unit $\eta \times \phi$ area and unit $\mu$ (number of interactions per bunch crossing) as a function of the distance (in BCID, bunch crossing identifier, in $25 \mathrm{~ns}$ units) from the beginning of the bunch train in the ATLAS EM calorimeter for $0.8<|\eta|<1.0$ [3].

In 2016, data have been collected using a random trigger around the colliding bunch, 4 samples have been recorded for each incoming LAr analog signal. In 2017 and 2018, 32 samples were recorded and the readout delay has been shifted in steps of about $3 \mathrm{~ns}$. In both ways, a combination of multiple events provides a very accurate measurement of the pulse shape. Figure 3a shows an example from the 2016 measurement.

A precise pluse shape measurement helps improving the calorimeter energy response.

When the cell energy is computed, a correction is applied to account for the shape difference between an ionization pulse and a pulse from charge injection. Pulse shapes measured in test beams were previously used to derive such corrections. The new measurement from collision data reduces the systematic uncertainties on the computed energy.

Another application for this measurement is the improvement of the LAr baseline correction.

The LAr bipolar pulse shape would lead to perfect cancelation of all signals if they are uniformly distributed in time. At the beginning of a bunch train the distribution is not uniform and thus no cancelation occurs. This leads to the average energy from PileUp being nonzero at the beginning of the train, as shown in Figure $3 b$.

The baseline correction is applied to remove this effect. Inputs to its derivation include the pulse shape per layer and cell in $\eta^{4}$ : an improved knowledge of the pulse shape causes a visible reduction of the residual systematic. In Figure $3 b$, the new correction (in red) is shown to describe the uncorrected data (black) better than what was previously available (blue).

\section{LAr operation and data quality}

A smooth running of the LAr Calorimeter has been achieved thanks to a continuous effort by a dedicated operation team.

A set of software tools has been put in place for online monitoring of the detector status, as well as a set of observables such as noise levels, trigger rates, timing and others, wich would later be used to assess data quality.

\footnotetext{
${ }^{4}$ Symmetrized in $\phi$.
} 


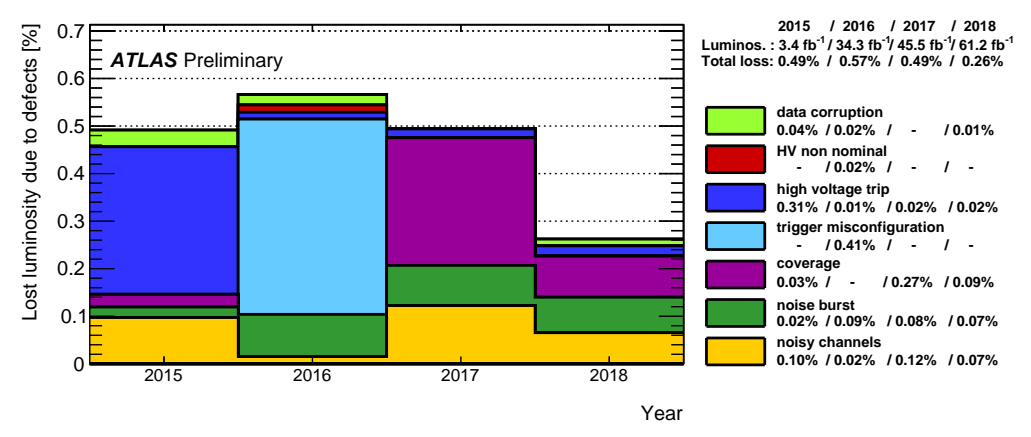

(a)

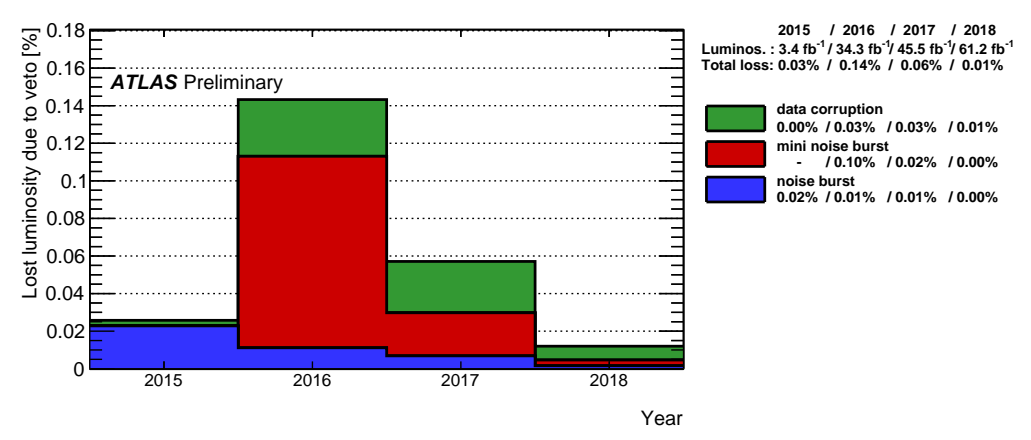

(b)

Figure 4: LAr data quality inefficiency in Run 2 [3]. a) Luminosity block defect rejection. b) Time veto rejection.

The monitoring has also been complemented by automatic recovery procedures. To give an example, an algorithm named LAr Trigger Tower Noise Killer treats noisy trigger towers by finding the calorimeter cell responsible for the noise and disabling it.

Once the data are recorded, a dedicated sample is used to assess the data quality, through a combination of automatized algorithms and human inspection. A detailed discussion of data quality procedures in ATLAS can be found in [5].

For the LAr Calorimeter, data quality operation consists in masking of noisy calorimeter cells ${ }^{5}$ and vetoing time periods affected by issues such as noisy events or corrupted data.

Two types of time veto are implemented: when the affected time period is less than 1 minute the exact time window is vetoed, longer lasting issues are removed by assigning a defect to whole luminosity blocks ${ }^{6}$.

Figure 4 shows the data loss per year due to each type of time veto. The total data quality inefficiency amounts to less than 1\%. In addition, a clear improvement is visible with the progressing of Run 2.

\subsection{Noise bursts}

One contribution to the data quality inefficiency comes from bursts of coherent noise occurring in the LAr Calorimeter. Two types are distinguished: the noise bursts, extending over wide regions

${ }^{5}$ The energy is extrapolated from neighbouring cells.

${ }^{6}$ One luminosity block corresponds to about 1 minute of data taking. 
and lasting between $1 \mu \mathrm{s}$ and $1 \mathrm{~ms}$, and the mini noise bursts, involving only a few cells from a single FEB and lasting less than $10 \mu \mathrm{s}$.

Both types of noise bursts cause signals with poor quality factors, affect the noisy cell masking procedure and make for a poor calorimeter clusters' energy reconstruction.

Noise bursts are treated by vetoing a time window around the noisy events: the associated data loss can be seen in Figure 4a (green), 4b (blue) for noise bursts and in Figure 4b (red) for mini noise bursts. Noise bursts cause permille level data quality inefficiency throughout Run 2, while the effect of mini noise bursts has been reduced from $0.1 \%$ in 2016 to negligible in 2018. These improvements are the result of a better software identification as well as high voltage tuning, which helps reducing the frequency of mini noise bursts occurrence.

\section{Conclusion}

The ATLAS LAr Calorimeter provided very high performances in Run 2. Data taking efficiency larger than $99 \% \%^{7}$, as well as good energy and time resolution, have been achieved. This has been made possible by a constant effort in improving the system's performance, in order to cope with increasingly difficult data taking conditions.

The LHC Run 2 ended in December 2018 and the ATLAS detector Phase I upgrade is ongoing since the beginning of 2019. A complete description of the LAr Calorimeter upgrade is beyond the purpose of this contribution, the main feature consists of a new trigger scheme, with the aim of keeping the Level-1 trigger thresholds sufficiently low, despite the higher PileUp environnement [6].

Studies for the HL-LHC upgrade (Phase II) are also progressing, a detailed description can be found in [7].

\section{References}

[1] ATLAS Collaboration, Luminosity public results, https://twiki.cern.ch/twiki/bin/view/AtlasPublic/LuminosityPublicResultsRun2.

[2] ATLAS Collaboration, The ATLAS Experiment at the CERN Large Hadron Collider, JINST 3 (2008) S08003

[3] ATLAS Collaboration, LAr public plots, Run 2, https://twiki.cern.ch/twiki/bin/view/AtlasPublic/LArCaloPublicResults2015.

[4] ATLAS Collaboration, Electron and photon performance measurements with the ATLAS detector using the 2015-2017 LHC proton-proton collision data, JINST 14 (2019) P12006

[5] ATLAS Collaboration, ATLAS data quality operations and performance for 2015-2018 data-taking, submitted to JINST, arXiv:1911.04632

[6] ATLAS Collaboration, ATLAS Liquid Argon Calorimeter Phase-I Upgrade Technical Design Report, ATLAS-TDR-022, https://cds.cern.ch/record/1602230/

[7] ATLAS Collaboration, Technical Design Report for the Phase-II Upgrade of the ATLAS LAr Calorimeter, ATLAS-TDR-027, https://cds.cern.ch/record/2285582

${ }^{7}$ Not to be confused with the $89 \%$ efficiency of the ATLAS detector as a whole 